\title{
Détermination expérimentale de l'efficacité de coalescence
}

\author{
par Baudouin Lismonde
}

du Laboratoire des Ecoulements Géophysiques et Industriels, Grenoble

\section{INTRODUCTION}

\subsection{Contexte de la recherche}

Une dispersion liquide-liquide dans un écoulement turbulent voit sa granulométrie commandée par les phénomènes de coalescence et les phénomènes de morcellement. En se limitant aux seuls processus de coalescence (Allan et al. [1962], Liem et al. [1974]), on peut dire que la fusion entre deux gouttes résulte de deux mécanismes successifs : d'une part, le rapprochement de deux gouttes avec une certaine fréquence d'occurence et une certaine force de rapprochement imposé par la structure hydrodynamique de l'écoulement (turbulence, cisaillement...), d'autre part, l'amincissement progressif du film séparant les deux gouttes, ou drainage, régi par les propriétés physico-chimiques des fluides et des interfaces (viscosité volumique, viscosité surfacique élongationnelle...). Comme les forces de rapprochement des gouttes sont fluctuantes, la fusion aura lieu ou non suivant que le drainage aura eu le temps de s'effectuer ou non. Ce deuxième mécanisme est essentiellement le même en écoulement turbulent et laminaire (Mariaux [1990]).

Soit un nombre $n$ de collisions à deux globules. On appelle efficacité de coalescence, ou taux de fusion, le rapport $E$ du nombre $n_{f}$ de fusions, à un nombre de référence $n_{c}$ de collisions. Cette efficacité dépend du modèle de référence retenu. Le modèle le plus simple consiste à prendre comme nombre $n_{c}$ de collisions le nombre de chocs en l'absence d'interaction hydrodynamique entre gouttes. On pourrait aussi tenir compte des interactions proches et modifier le nombre de collisions effectives. Bien entendu, la donnée de l'efficacité de coalescence doit être associée au modèle de référence retenu.

Pour bien comprendre l'intérêt d'une telle détermination, il faut savoir que cette efficacité est souvent totalement ignorée et que les codes ordinaires de calculs diphasiques peuvent adopter une valeur numérique de 1 alors que nos expériences fournissent des valeurs de l'ordre de $10^{-4}$ dans le cas de gouttes d'huile dans l'eau.

Les modèles physiques des écoulements turbulents diphasiques permettent de prédire le cisaillement local responsable de la rencontre de deux globules, mais il faut ajouter un scalaire traduisant la probabilité de fusionnement (Mariaux [1990], Thomas [1981]). Cette probabilité peut être calculée par d'autres modèles physiques compliqués faisant appel aux propriétés des surfactants (Traykov et al. [1977], Ivanov [1980], Zinchenko [1984], Muralidhar et al. [1988], Danov et al. [1994]), ou bien, et c'est la méthode que nous proposons, cette probabilité peut être déterminée expérimentalement pour une dispersion donnée.

\subsection{Objectif de l'étude}

Plutôt que d'effectuer des mesures délicates des propriétés physico-chimiques d'un liquide dispersé dans un autre, typiquement du pétrole dispersé dans de l'eau, et ensuite d'injecter ces mesures dans un modèle de drainage, on propose une méthode expérimentale directe d'évaluation de l'efficacité de coalescence en écoulement laminaire basée sur une mesure à l'aide d'un appareil, le coalesceur

An experimental method is presented to determine the coalescence efficiency of a liquid-liquid dispersion. A laminar Taylor-Couette flow is choised as reference flow.

The Sauter diameter of the dispersion is measured with a turbidimetric probe. The coalescence efficiency is deduced of a physical code.

The hydrodynamic diffusivity of dispersed population is calculed with complementaries video experiments. 
de Couette, suivie d'une analyse s'appuyant sur un modèle physique intégré dans des logiciels simples d'emploi.

Le modèle physique est construit à l'aide des résultats accessibles dans la littérature scientifique et s'appuie, pour le reste, sur une expérimentation particulière.

\section{DÉTERMINATION DE L'EFFICACITÉ DE COALESCENCE}

\subsection{Obtention expérimentale du diamètre de Sauter et de son évolution temporelle}

Nous allons décrire les manipulations et les calculs qui permettent d'accéder au diamètre de Sauter d'une dispersion préparée sur place. On pourrait, bien sûr, injecter directement dans le coalesceur une dispersion dont on voudrait étudier les caractéristiques.

On fabrique une dispersion de gouttes dans de l'eau dégazée (grâce à une pompe à vide) en injectant un débit constant d'huile dans un écoulement d'eau en régime permanent. La fraction volumique de l'huile est réglable. Elle est égale au rapport entre les débits d'huile et d'eau à l'injection, en l'absence de glissement entre les deux phases. Les deux débits sont indépendants et mesurés par des rotamètres qui fonctionnent uniquement avec de l'eau, comme on peut s'en assurer sur le schéma.

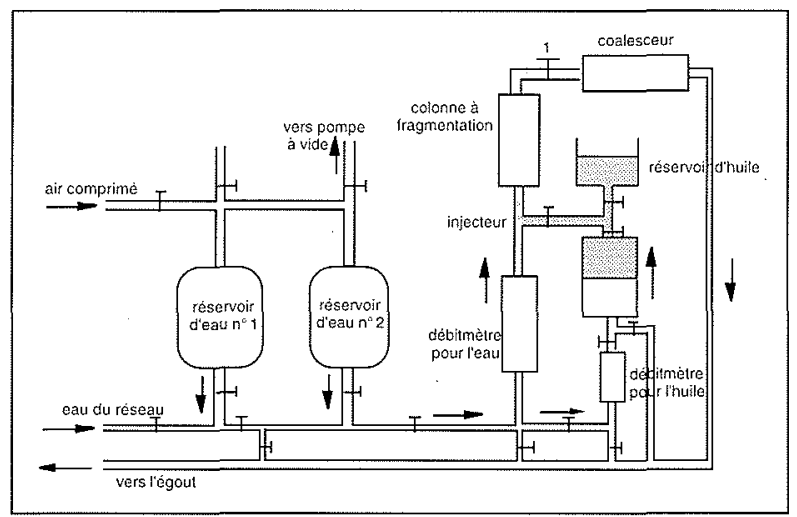

1. Schéma de l'installation. L'eau est envoyée des réservoirs vers le coalesceur par deux chemins en parallèle. Dans la deuxième branche, l'eau pousse l'huile dans l'injecteur.
Ensuite, on fragmente les gouttes d'huile dans une colonne entrecoupée d'une succession de 10 diaphragmes de $4,8 \mathrm{~mm}$ d'ouverture. Le rapport des débits de l'eau et de l'huile est imposé par la valeur de la fraction volumique d'huile que l'on veut obtenir, mais l'échelle des débits est commandée, quant à elle, par le diamètre souhaité des gouttes à la sortie de la colonne de fragmentation. Il est clair, sans rentrer dans les détails des mécanismes physiques, que la taille moyenne obtenue à l'issue du passage dans les 10 diaphragmes est d'autant plus petite que la vitesse est plus grande. Si l'on veut éviter de faire trop varier les débits, on peut aussi jouer sur le diamètre des diaphragmes. A débit constant, la taille des inclusions diminue quand le diamètre des diaphragmes diminue.

Cette dispersion est aussitôt injectée dans le coalesceur de Couette constitué de deux cylindres concentriques de diamètres voisins et de $200 \mathrm{~mm}$ de longueur, La distance entre les cylindres est de $1 \mathrm{~mm}$. On peut faire tourner au choix le cylindre intérieur ou le cylindre extérieur. La vitesse est comprise entre 1 et $150 \mathrm{rd} / \mathrm{s}$. L'axe des cylindres est situé dans un plan horizontal. Le cylindre intérieur est en acier inoxydable, alors que le cylindre extérieur est en verre afin de permettre des mesures optiques. Le fluide diphasique circule à travers l'espace annulaire et la fermeture de la vanne 1 interrompt le mouvement axial du fiuide. Il reste soit un mouvement tangentiel cisaillé, dit écoulement de Couette circulaire (Couette [1880]), d'où le nom de l'appareil, soit un écoulement plus compliqué constitué par un empilement de tores en rotation alternée (Couette [1880], Taylor [1923]).

Turbidimètre laser. La mesure du diamètre de Sauter peut commencer. Elle est effectuée par un turbidimètre laser. Le principe de cet appareil est classique (Langlois et al. [1954], Laughlin et al. [1973], Véteau [1981], Mariaux et Achard [1988], Mariaux [1990]), mais l'application à l'écoulement de Couette circulaire est originale. Un faisceau lumineux traversant le mélange diphasique est absorbé partiellement par les gouttes rencontrées sur le trajet. Ce faisceau est réfléchi sur le cylindre intérieur en acier inoxydable poli et revient tomber sur une photodiode. La comparaison entre les intensités lumineuses recueillies en l'absence et en présence des gouttes permet de remonter à l'aire interfaciale comme l'a montré Véteau [1981]. En effet, plus les gouttes sont petites et plus, à concentration égale, elles opacifient le liquide car la surface apparente totale des gouttes est inversement proportionnelle à leur taille moyenne.

La loi d'absorption est donnée par $\ln \left(\frac{\Phi_{0}}{\Phi}\right)=\frac{\Gamma(t) e}{4}$

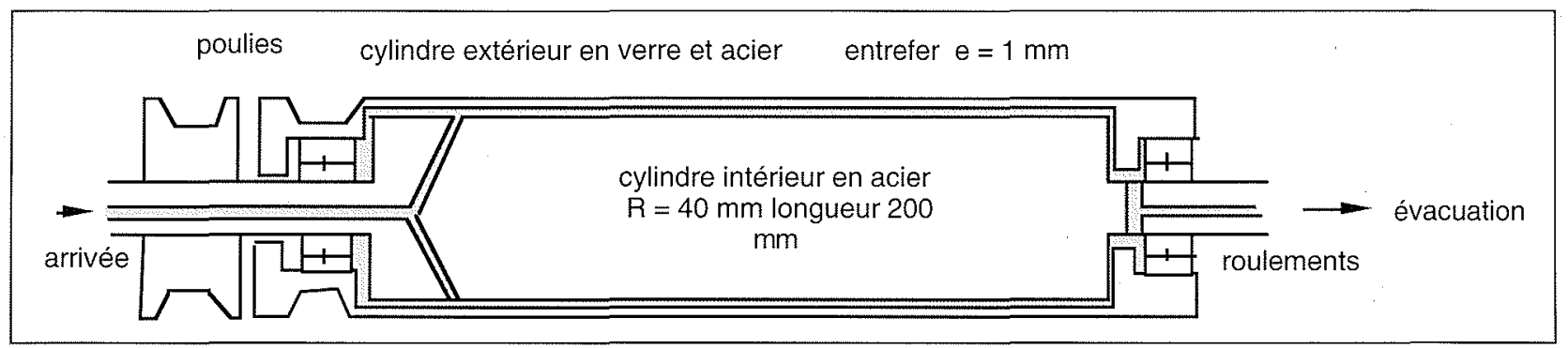

2. Le coalesceur de Couette. On peut faire tourner au choix le cylindre intérieur ou le cylindre extérieur. 


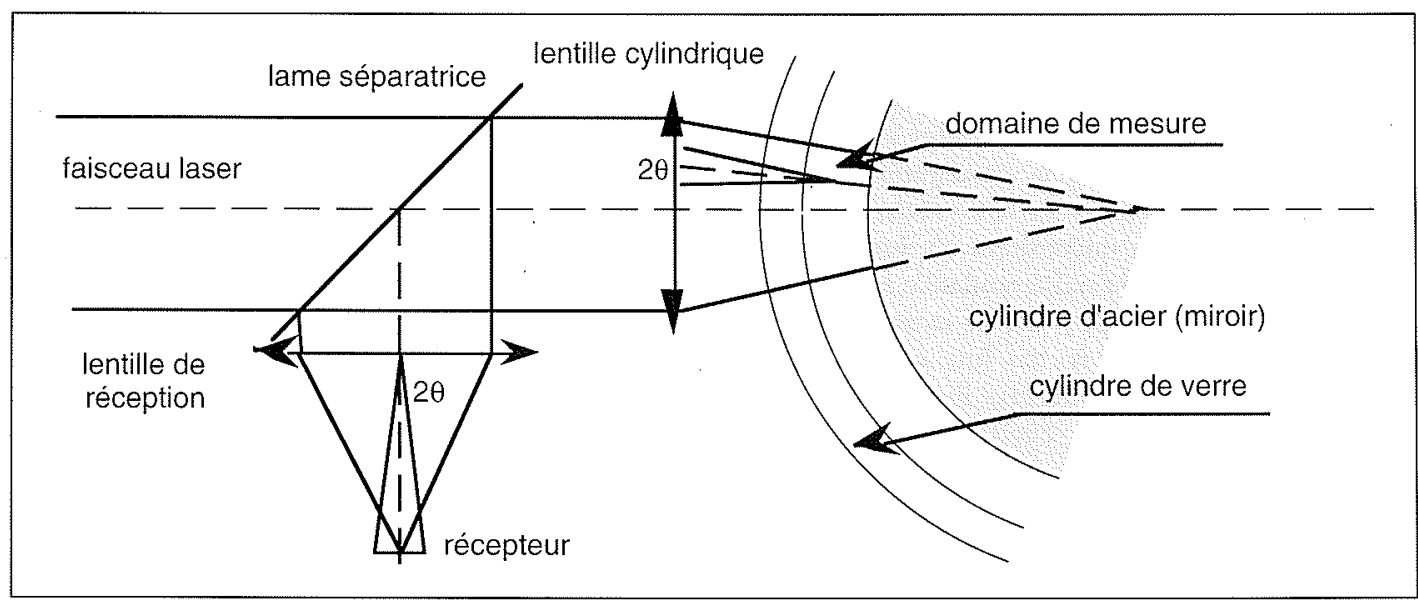

3. Le turbidimètre laser. Le faisceau laser reste perpendiculaire aux parois, il est atténué deux fois par le mélange diphasique.

avec $\Phi_{0}$ intensité du faisceau non atténué, $\Phi(t)$ entensité du faisceau atténué, $\Gamma(t)$ aire interfaciale volumique $\left(\mathrm{m}^{-1}\right)$, e épaisseur de mélange traversé.

La présence de plusieurs surfaces optiques sur le trajet lumineux complique un peu le calcul de l'intensité lumineuse abordant le fluide (Kibboua [1994]).

Effet de masque. Par rapport au montage classique, un problème nouveau est apparu. Dans le calcul de l'absorption d'un faisceau lumineux, les particules sont supposées aléatoirement disposées à petite échelle (distribution de Poisson). Cette propriété est à peu près réalisée dans notre écoulement mais la présence du miroir introduit dans son voisinage une corrélation égale à un et rompt la structure de la répartition poissonnienne. Nous avons appelé cet effet, l'effet de masque. On peut en donner une interprétation très simple dans le cadre de l'optique géométrique. Le faisceau lumineux qui a rencontré une particule présente une lacune d'intensité en arrière de la particule (dans son ombre). Cette ombre est réfléchie dans le miroir et le faisceau qui revient est fortement atténué. Le faisceau rencontre alors une deuxième fois la particule. La deuxième atténuation est donc moins grande que la première. Cela équivaut à dire qu'une particule n'intervient pas deux fois dans l'absorption mais un peu moins $(n<2)$. Heureusement, il se trouve que la longueur de l'ombre calculée en optique physique est petite devant l'entrefer et par ailleurs, les particules ont une concentration nulle au voisinage immédiat du cylindre intérieur. Néanmoins, nous avons été obligé de faire une correction systématique basée sur l'optique physique (Nussenzveig [1965], Kibboua [1994]) pour tenir compte de ce phénomène, correction traduite par la relation :

$$
n=2-\int_{0}^{L / 2} \frac{\alpha}{\alpha_{m}}(1-2 e x / L)^{2} \mathrm{~d} x \text { avec } L=\frac{1}{2}\left(\frac{\pi}{\lambda}\right)^{1 / 3} D^{4 / 3}
$$

Dans cette expression $\alpha$ est la concentration locale d'huile, $\alpha_{m}$ la concentration moyenne, $x$ la coordonnée radiale sans dimension et $\mathrm{L}$ la longueur de l' « ombre», $D$ le diamètre de la goutte et $\lambda$ la longueur d'onde de la lumière. Nous n'avons pas vérifié expérimentalement la validité de notre correction. Mais l'examen des résultats montre que le facteur de correction est très petit et donc qu'il n'est pas nécessaire de connaître cette correction avec une grande précision.

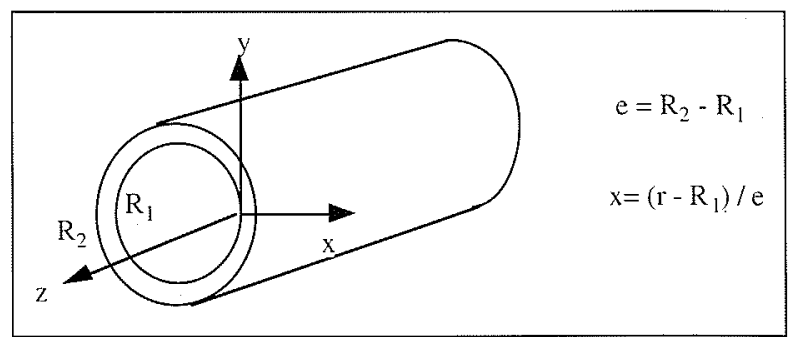

4. Le repère local avec les coordonnées sans dimensions.

Diamètre de Sauter. Le turbidimètre délivre une tension électrique dont on peut mesurer la valeur au cours du temps. Après calcul, cette tension fournit l'aire interfaciale dont on peut ainsi suivre l'évolution. Pour des valeurs normales des tensions superficielles et les valeurs des cisaillements obtenus dans le coalesceur, les gouttes restent sphériques et l'aire interfaciale volumique $\Gamma$ est reliée au diamètre de Sauter $D_{s}$.

$$
D_{s}=\left[\sum_{i=1}^{n} D_{i}^{3}\right] /\left[\sum_{i=1}^{n} D_{i}^{2}\right] \text { et } D_{s}=\frac{6 a}{\Gamma}
$$

On a constaté, en faisant les mesures, que la courbe d'évolution du diamètre de Sauter présentait une forte irrégularité dans le cas du cylindre extérieur tournant alors que cette courbe était plus lisse dàns le cas du cylindre intérieur tournant. Il est bien connu que ces deux configurations sont très différentes. La première, cylindre extérieur tournant, est stable et l'écoulement reste un simple écoulement cisaillé, du moins tant que l'écoulement reste laminaire, alors que le cylindre intérieur tournant conduit rapidement à l'apparition d'une rupture de symétrie se traduisant par les tourbillons de Taylor (Stuart [1958], Coles [1965], Cognet [1984], Marcus [1984], Andereck et al. [1986], Nagata [1986], [1988]). La régularité de la courbe d'évolution du diamètre de Sauter dans le cas du 
cylindre intérieur tournant vient sans doute du confinement des gouttes d'huile qu'engendrent les anneaux de Taylor. On verra plus loin que les gouttes se rassemblent au centre des anneaux, les empêchant de migrer vers les extrémités du coalesceur. En revanche, dans la configuration sans anneau de Taylor; on peut montrer que les couches limites créées aux bouts du coalesceur engendrent un mouvement lent tridimensionnel de pompage qui a tendance à ramener les gouttes aux extrémités, près de l'axe de rotation. Cette constatation nous a donc incité à ne pas fuir, au contraire, la formation d'anneaux de Taylor, au prix il est vrai d'une complication dans la prévision des répartitions radiales de gouttes.

\subsection{Modèle numérique d'évolution du diamètre de Sau- teur}

En partant d'une granulométrie initiale des gouttes obtenues expérimentalement grâce aux mesures décrites plus loin en 3-2, on peut calculer l'évolution de la granulométrie par une équation de bilan de population (Müller [1928], Ramkrishna et al. [1973], Muralidhar et al. [1986]). Le principe du calcul utilisé ici est simple (code smoluc pour l'écoulement de Couette ou smolut pour les anneaux de Taylor). On considère un certain point dans l'entrefer. En ce point règne une certaine concentration en huile et un certain cisaillement. La répartition granulométrique en ce point peut évoluer en fonction du temps mais, et c'est là une hypothèse fondamentale, la granulométrie est la même d'un point à un autre (elle dépend du temps mais elle est homogène spatialement). Cette propriété a été vérifiée expérimentalement par comptage de gouttes. Elle se comprend si on admet que le temps caractéristique de diffusion d'une goutte dans l'entrefer est plus petit que celui nécessaire à modifier la granulométrie.

On peut donner une estimation du temps de redistribution de la granulométrie $t=e^{2} / G D^{2} \alpha_{m}$ où $G$ est le cisaillement et $\alpha_{m}$ la fraction volumique dispersée. On trouve un temps de redistribution de l'ordre d'une seconde pour le coalesceur, ce qui est faible par rapport au temps d'évolution de la granulométrie, en général de l'ordre de plusieurs minutes.

Pendant un intervalle de temps donné, on calcule au point considéré, et pour chaque classe de volume de gouttes, le nombre de rencontres avec les gouttes des autres tailles. Pour l'écoulement de Couette, nous avons utilisé l'expression de Smoluchowski [1917]:

$$
n_{12}=\frac{8}{3} N_{R 1} N_{R 2}\left(R_{1}+R_{2}\right)^{3} \frac{G}{2}
$$

où $N_{R 1}$ et $N_{R 2}$ sont le nombre de gouttes de volume $V_{1}$ et $V_{2}$ par unité de volume, associés aux rayons $R_{1}$ et $R_{2}$ et $G$ le cisaillement moyen (en $\mathrm{s}^{-1}$ ).

Pour l'écoulement avec anneaux de Taylor, nous avons généralisé l'expression précédente à un écoulement quelconque :

$$
\begin{aligned}
n_{12}= & \frac{8}{3} N_{R 1} N_{R 2}\left(R_{1}+R_{2}\right)^{3} \varepsilon f(\eta) \\
& \text { avec } f(\eta)=\frac{1}{2} \int_{0}^{2 \pi} \frac{\left[(\eta-1) \sin ^{2} \theta+1\right]^{3 / 2}}{\left[(\eta-1) \sin ^{2} \theta+2+\eta\right]^{1 / 2}} \mathrm{~d} \theta
\end{aligned}
$$

Dans cette expression $\varepsilon$ est le plus grand des taux de déformations de l'écoulement (dans le repère local prin- cipal des déformations) et $\eta$ est le rapport entre la déformation principale de valeur médiane (positive sinon on retoume le repère principal) et la plus grande des déformations. La fonction $f$ varie entre 1 et 1,814 pendant que $\eta$ varie de 0 à 1 . Le fluide est supposé incompressible. Dans le cas d'un écoulement simplement cisaillé $f(0)=1$ et $\varepsilon=G / 2$.

Que l'expression de Smoluchowski (ou généralisée) soit valable n'est pas du tout évident a priori. En effet, elle suppose les inclusions réparties au hasard dans l'écoulement. Or la trajectoire circulaire de l'écoulement de Couette devrait amener une certaine raréfaction des rencontres par rapport à celles fournies par le hasard puisqu'une inclusion balaye un certain tore qui ne contient plus qu'une seule inclusion. Cet effer de sillage (corrélation entre les positions des inclusions) est contrebalancé efficacement par la redistribution due à la diffusivité que nous avons évoquée au dessus. Finalement l'approximation de répartition au hasard est bien vérifiée.

On en déduit en chaque point le nouveau nombre de gouttes pour chaque classe de taille. Comme on peut montrer que le nombre de rencontres est proportionnel au cisaillement local et au carré de la concentration locale, il est facile d'étendre le bilan de population d'un point particulier à l'ensemble de l'entrefer et cela que l'écoulement soit celui de Couette ou celui avec anneaux de Taylor, à condition bien sûr de connaître la répartition des concentrations et le cisaillement local (cf. chapitre 3). C'est le facteur $F$ ( $\varepsilon$ et $\eta$ dépendent des coordonnées $x$ et $z$ ):

$$
F=\frac{2}{\alpha_{m}^{2} G} \int_{\text {plan méridien } x z} \alpha^{2} \varepsilon f(n) \mathrm{d} x \mathrm{~d} z
$$

La nouvelle granulométrie fournit un nouveau diamètre de Sauter (homogène dans tout l'entrefer).

\subsection{Comparaison numérique et expérimentale de l'évo- lution du diamètre de Sauter et détermination du taux de fusion}

On peut donc déterminer au cours du temps l'évolution du diamètre de Sauter de deux façons indépendantes, d'une part, expérimentalement en mesurant l'atténuation lumineuse et d'autre part, numériquement. La comparaison des dérivées (sans dimension) par rapport au temps du diamètre de Sauter nous fournit l'efficacité de coalescence moyenne dans l'entrefer. On voit en effet sur l'expression ci-dessous, qui résulte du bilan de population, que cette dérivée est proportionnelle à l'efficacité de coalescence $E$, à la condition que cette efficacité soit supposée la même pour toutes les rencontres.

$$
\begin{aligned}
& \frac{1}{G D_{s}} \frac{d D_{s}}{d t}=\frac{4}{3} E F\left[\frac { 1 } { 2 } \sum _ { j = 1 } ^ { i - 1 } N _ { j } N _ { i - j } \left(R_{j}\right.\right.\left.+R_{i-j}\right)^{3}- \\
&\left.-\sum_{j=1}^{p} N_{i} N_{j}\left(R_{i}+R_{j}\right)^{3}\right] \times \\
& \times \frac{\sum_{i=1}^{p}\left[R_{i}^{3} \sum_{i=1}^{p} N_{i} R_{i}^{2}-R_{i}^{2} \sum_{i=1}^{p} N_{i} R_{i}^{3}\right]}{\left[\sum_{i=1}^{p} N_{i} R_{i}^{3}\right]\left[\sum_{i=1}^{p} N_{i} R_{i}^{2}\right]}
\end{aligned}
$$


$N_{i}$ et $N_{j}$ sont les nombres de gouttes par unité de volume pour les classes de gouttes de volume $V_{i}$ et $V_{j}$ (en progression arithmétique) associés aux rayons $R_{i}$ et $R_{j}$, $p$ est le nombre de classes de gouttes.

L'efficacité de coalescence que nous mesurons est une grandeur moyennée qui correspond à une dispersion donnée, de taille moyenne et pour un cisaillement donnés.

Les mesures nous fournissent des taux d'efficacité de coalescence qui varient suivant les huiles mesurées de $1.10^{-4}$ à $5 \cdot 10^{-4}$. La viscosité des huiles (variant dans un rapport 10) ne joue pratiquement aucun rôle. C'est la présence de surfactant que nous n'avons pas cherché à éviter qui doit expliquer ce résultat. Si l'on compare ces valeurs à celles trouvées dans d'autres expériences, elles sont un peu supérieures (Mariaux [1990]).

Le nombre de rencontres par seconde a été obtenu en supposant que les gouttes n'interagissaient pas. On a déjà vu plus haut que cette hypothèse est en fait une convention et que l'efficacité de coalescence est associée à la convention faite. Si on voulait tenir compte des interactions proches afin que le taux de fusion résulte uniquement du drainage du film, il suffirait de modifier le modèle numérique. Mais il nous semble préférable de foumir une valeur du taux de fusion qui soit indépendante de la qualité d'un modèle d'interaction proche.

\section{RÉPARTITION RADIALE DES GOUT- TES}

Au niveau de la modélisation du bilan de population (cf. 2-2). des caractéristiques de l'écoulement comme la répartition radiale de concentration qui résulte elle-même de la diffusivité (ou dispersivité) due aux interactions hydrodynamiques n'ont pu être déterminées à partir des données disponibles. Des mesures ont certes fourni des valeurs de diffusivités (Eckstein et al. [1977], Leighton et al. [1987], Nadim [1988], Koch [1989], Acrivos et al. [1992], Chow et al. [1994]), mais il était important d'obtenir directement le profil radial sans passer par les valeurs numériques de diffusivité obtenues dans d'autres expériences, surtout pour la configuration avec les cellules de Taylor. Nous avons donc étudié cette répartition radiale, ce qui nous a obligé à développer des mesures et des modèles d'exploitation.

\section{3,1 Détermination expérimentale des répartitions radia-} les de concentration en gouttes

Nous avons mis au point une technique de prise de vue de l'écoulement pour analyser la répartition radiale des gouttes. Ce travail ne fait pas partie de l'utilisation normale du coalesceur. Il a été exigé par le fait que nous n'avions pas de données utilisables directement. La technique utilisée est très lourde puisqu'elle consiste à analyser des images prises au microscope. Un éclateur à arc au Xénon fournit des éclairs très brefs de quelques microsecondes. Une caméra CCD installée sur un microscope enregistre l'image (technique de l'open-flash) figée par la rapidité de l'éclair. Sur les images, le diamètre est directement mesurable grâce à une table à digitaliser. On peut accéder à la position de la goutte dans l'entrefer grâce à une technique originale. On utilise l'image réfléchie de la goutte dans le cylindre intérieur qui sert de miroir. La mesure de

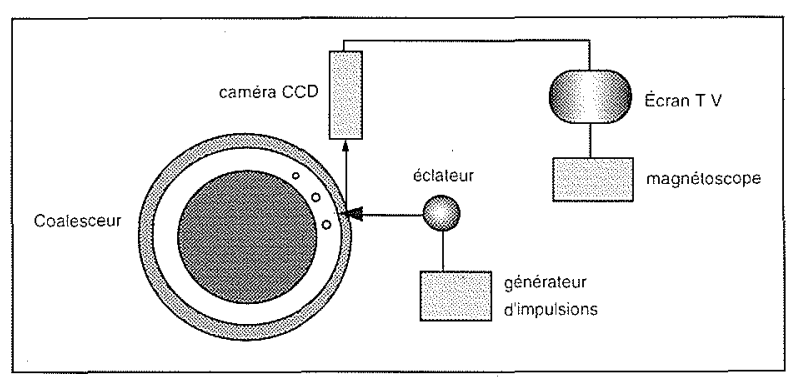

5. Analyse vidéo des images. La lumière est fournie par un éclateur rapide, la caméra $\mathrm{CCD}$ enregistre les images figées.

la distance entre l'image et la goutte donne la distance au cylindre intérieur avec une bonne précision. On obtient donc la fraction volumique, la répartition en taille et la répartition radiale des gouttes.

L'analyse de l'image revient à apparier les gouttes, à éliminer les gouttes non appariées car situées à la périphérie de l'image, à mesurer pour chaque couple le diamètre et la distance (position radiale).

L'analyse statistique des nombreuses images analysées consiste à classer les gouttes par diamètre et à dénombrer chaque classe. Pour accéder à la concentration, il faut déterminer le volume d'huile par image en tenant compte d'un effet de bord complexe qui élimine les gouttes non appariées.

Cette analyse est très complète, malheureusement, la lourdeur de l'exploitation des images (à la table à digitaliser) interdit une utilisation systématique de cette méthode.

\subsection{Modèle numérique de répartition radiale de concen- tration en gouttes}

L'étude du comportement d'une goutte isolée montre qu'elle est soumise à des forces systématiques et des forces aléatoires. Les forces systématiques comprennent 4 forces : la répulsion de paroi, calculée analytiquement par Chan et Leal [1979] dans le cas de l'approximation des écoulements rampants, la force due à la courbure des lignes de courant calculée aussi par Chan et Leal [1979], la force due au gradient de pression (poussée d'Archimède) qui tend à repousser les gouttes vers le cylindre intérieur et la force de pesanteur qui contrairement aux autres ne possède pas de symétrie de révolution. Les forces aléatoires résultent des interactions hydrodynamiques entre les différentes gouttes. Alors que pour les forces systématiques, il existe des expressions analytiques simples d'emploi (dans le cas de gouttes uniques et dans l'approximation des écoulements rampants), en revanche, dans le cas des interactions hydrodynamiques, il n'existe pas d'expression utilisable même dans le cas des interactions à deux gouttes. Les seules théories disponibles concernent des interactions à deux sphères solides.

Pour les forces sytématiques, nous avons utilisé les expressions analytiques pour une goutte comme si elle était isolée et dans l'approximation des écoulements rampants alors que le nombre de Reynolds particulaire peut atteindre 10. Pour les forces aléatoires et en l'absence d'une théorie utilisable, nous avons admis que les interactions hydro- 
dynamiques se traduisaient par une agitation aléatoire assimilable à une diffusion macroscopique et régie par un tenseur de diffusivités inconnu.

La présence des gouttes influence en retour l'écoulement de base. On peut dire que les gouttes augmentent la viscosité efficace (qui donne la même dissipation visqueuse). Il en résulte aux endroits du maximum de concentration, une diminution locale du taux de cisaillement de l'écoulement. Nous avons retenu un comportement newtonien de la suspension et avons pris comme viscosité efficace l'expression d'Einstein [1906], [1911] valable dans un milieu infini car les concentrations sont faibles. Cette expression est plus représentative que celle de Taylor [1932] quand des surfactants contaminent les interfaces et les rigidifient. Les équations obtenues sont celles du «modèle à mélange » (Kibboua, Lismonde [1993]).

Nous avons alors élaboré un code incorporant la répartition en taille des gouttes dans l'entrefer et les vitesses induites par les forces dans l'approximation de Stokes. La répartition en taille est supposée uniforme, c'est-à-dire que la granulométrie est supposée la même dans tout l'entrefer. Cette hypothèse a déjà été utilisée pour le bilan de population. Ce code possède deux versions, l'une pour l'écoulement de Couette (Asco), l'autre pour l'écoulement en tourbillons de Taylor (Taylor). Dans les deux cas, le résultat est la répartition radiale et éventuellement axiale de la concentration des gouttes et le profil de vitesse associé. C'est un profil peu différent de celui obtenu en l'absence de gouttes puisque les concentrations sont supposées faibles. La diffusivité est inconnue. C'est la comparaison avec l'expérience qui permettra de la déterminer.

$\frac{\partial \alpha}{\partial t}+\frac{\partial}{\partial x}(\alpha u)+\frac{\partial}{\partial z}(\alpha w)=\frac{\partial}{\partial x}\left(\alpha D_{x} \frac{\partial \alpha}{\partial x}\right)+\frac{\partial}{\partial z}\left(\alpha D_{z} \frac{\partial \alpha}{\partial z}\right)(8)$

Dans l'étude de la répartition des concentrations, nous avons utilisé une équation de diffusion-convection en régime instationnaire (Lismonde [1989], Phillips et al. [1992]). On démarre avec une répartition quelconque et on attend qu'une solution asymptotique s'établisse.
3.3 Comparaison numérique et expérimentale sur la répartition radiale des gouttes et détermination de la diffusivité (ou dispersivité)

L'observation a montré que les gouttes se disposent préférentiellement vers le milieu des anneaux de Taylor et qu'il n'y a pas de ségrégation en taille, c'est-à-dire que la répartition granulométrique est indépendante de la position radiale.

La comparaison entre les profils mesurés et les profils résultant des codes permet de tester les lois de forces systématique d'une part et conduit à la détermination expérimentale de la diffusivité d'autre part.

Cas du cylindre extérieur tournant. On constate que dans le cas de l'écoulement de Couette, les lois retenues ne conviennent pas parfaitement. Les profils de concentration expérimentaux sont plus fortement décalés vers le centre que les profils obtenus par le code. Cela est sans doute dû à une surévaluation des forces de répulsion de paroi, forces qui, rappelons le, ne sont valables qu'à bas nombre de Reynolds alors que nous les avons utilisées jusqu'à des nombres de Reynolds voisins de 10 .

Dans l'exemple montré sur la figure, le facteur de correction à apporter à la force de répulsion de paroi serait de 0,5 .

La diffusivité ou dispersivité qui résulte des interactions hydrodynamiques entre les gouttes est la grandeur que l'on cherche à déterminer. Il est facile dans le code numérique d'introduire une diffusivité proportionnelle à la concentration locale de façon à reproduire au mieux, au sens des moindres carrés, le profil expérimental. On obtient ainsi dans notre cas une diffusivité de l'ordre de $7.10^{-8} \mathrm{~m}^{2} / \mathrm{s}$. Dans le cas du coalesceur de Couette avec le cylindre extérieur tournant et un écoulement à cisaillement pur, l'analyse dimensionnelle fournit une unité de diffusivité égale à $G R^{2} \alpha, G$ étant le cisaillement, $R$ le rayon moyen des gouttes et $\alpha$ la concentration locale (Leighton et Acrivos [1987]). L'expérience confirme cette analyse et fournit une diffusivité égale à $0,8 G R^{2} \alpha$.

Il est évident que cette expression ne peut pas être universelle, en effet, il n'y a pas que le cisaillement qui

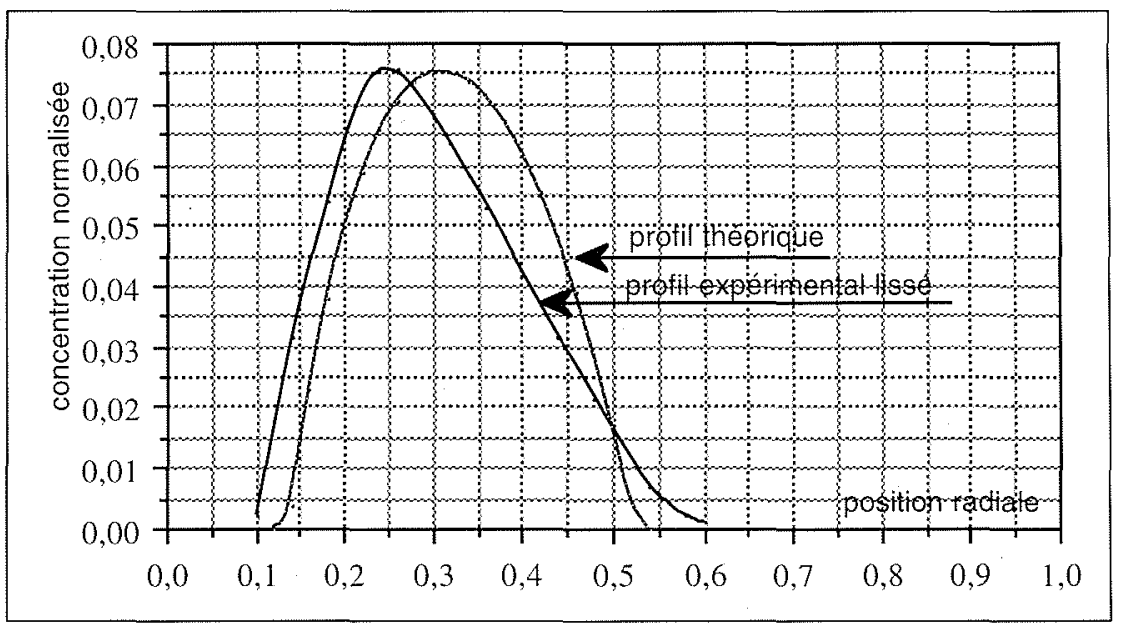

6. Comparaison entre l'expérience et le modèle numérique (pour une vitesse de rotation du cylindre extérieur de $400 \mathrm{t} / \mathrm{mn}$, un diamètre de Sauter de $106 \mu \mathrm{m}$ et une concentration moyenne de 1,96\%). L'étalement est ajusté au mieux, il reste un défaut de centrage. 


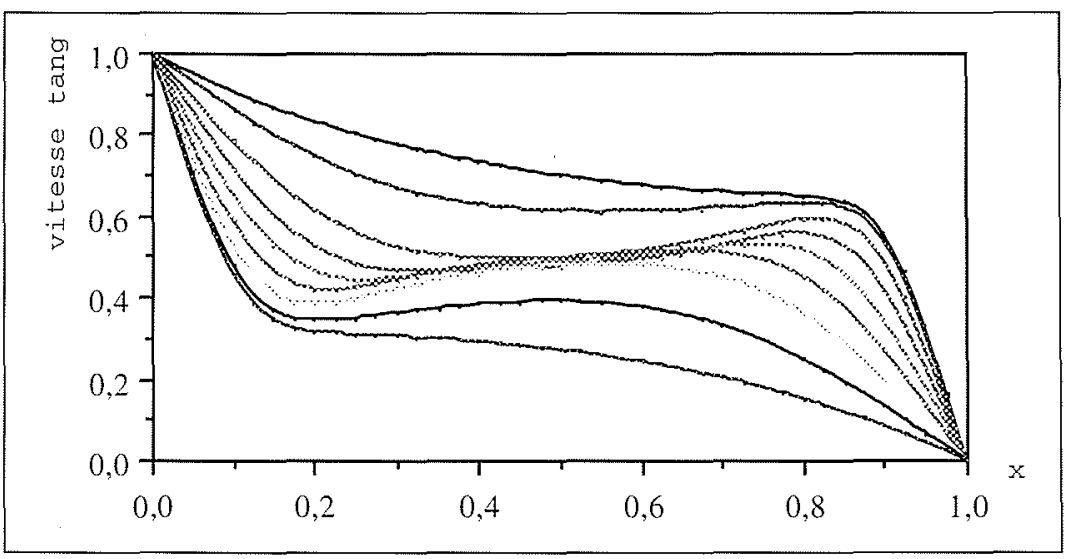

7. Profil des vitesses tangentielles aux anneaux de Taylor. Le gradient de vitesse dans la zone de présence des gouttes est plus petit que le cisaillement moyen.

contribue à cette diffusivité (comme dans la partie centrale d'un profil de Poiseuille par exemple). Mais dans notre expérience c'est bien sûr le cisaillement qui est le terme principal.

Cas du cylindre intérieur tournant. C'est le cas le plus intéressant comme on l'a vu plus haut car la mesure du turbidimètre est plus régulière. Les anneaux procurent un gradient de pression qui a tendance à ramener les gouttes sur l'axe circulaire interne du tore constitué par l'écoulement. De ce fait, la répartition radiale des gouttes est symétrique.

On constate que le maximum des concentrations est voisin de celui prévu théoriquement, il n'y a pas lieu de modifier la relation de répulsion de paroi. La diffusivité que l'on peut déduire de plusieurs expériences est $2,5 G R^{2} \alpha$.

Si on compare cette diffusivité avec celle obtenue dans le cas du cylindre extérieur tournant, elle est 3,1 fois plus grande. Ce résultat n'est pas très facile à expliquer puisque le cisaillement réel au cœur de la cellule de Taylor est plus faible, comme on peut le voir sur la figure 7 , que le cisaillement moyen.

\section{BILAN DE L'ÉTUDE, LE COALES- CEUR}

La méthode que nous proposons est tributaire des limitations imposées par les choix géométriques et cinématiques du coalesceur de Conette. Cela entraine les limitations suivantes sur la dispersion dont on veut mesurer l'efficacité de coalescence : la dimension d'une goutte doit être grande devant la longueur d'onde du rayonnement, l'éclairement d'une goutte est uniforme ce qui conduit à un diamètre du faisceau grand devant celui d'une goutte, l'optique de réception recuellle la partie transmise et aussi la partie diffractée par les gouttes, la concentration en goutte est assez faible pour éviter les problèmes de diffusion multiples.

Il reste à appliquer la méthode dans des conditions industrielles pour en tester la pertinence.

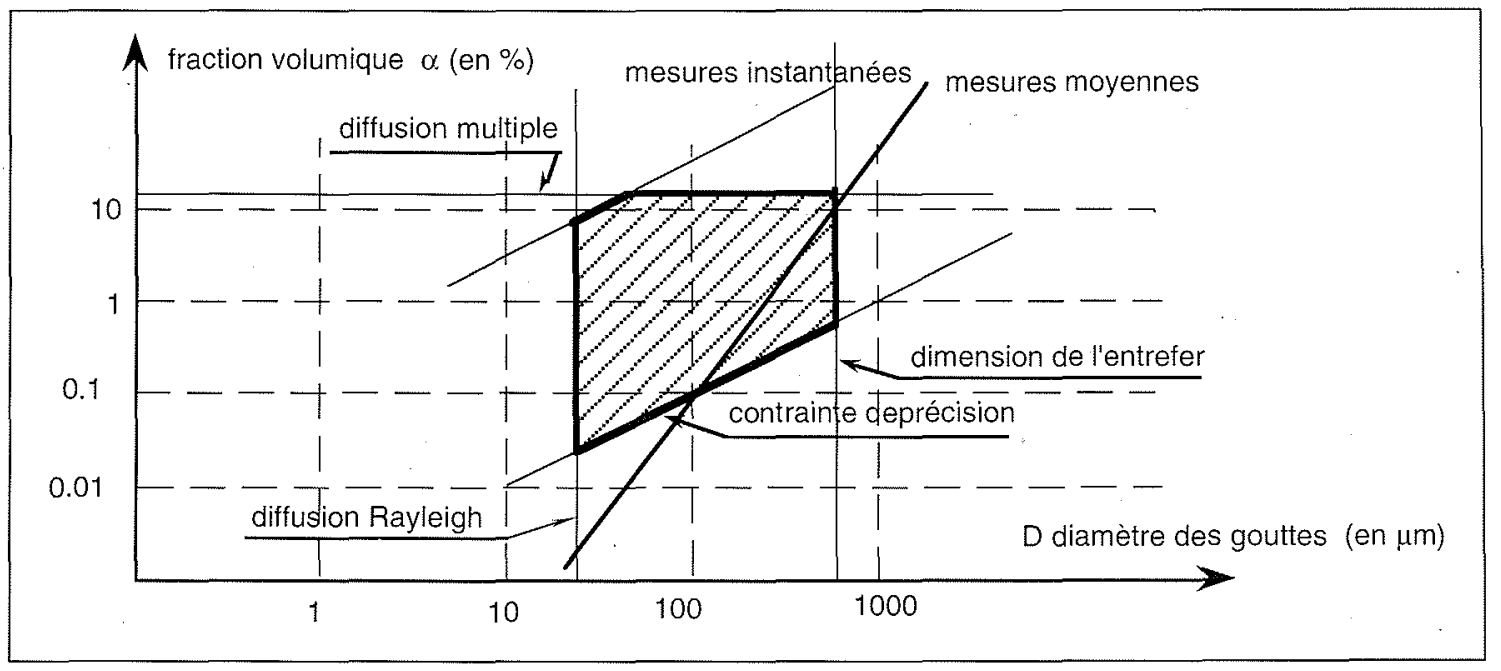

8. Plage des caractéristiques des dispersions mesurables (pour $e=1 \mathrm{~mm}$ et $\lambda=0,2 \mu \mathrm{m}$ ). Le coalesceur doit être adapté à la dispersion à mesurer. 


\section{Références}

AbBott J.R., Tetlow N., Graham A.L., Altobelli S.A., Fukushima E., Mondy L.A., Stephens T.S. (1991). Experimental observations of particle migration in concentrated suspensions: Couette How, J. Rheol. 35 p. 773.

Acrivos A., Batchel_or G.K., Hinch E.J. KOCH D.L., MAURI R. (1992). - Longitudinal shear-induced diffusion of spheres in a dilute suspension. J.F.M., 240, p. 65 I.

Allan R.S., MAȘON S.G. (1962). - Particle motions in shear suspensions. Part 1. coalescence of liquid drops in electric and shear fields. J. of Colloid and interface Sciences, vol. 17, pp. 383-408

ANDERECK C.C., Liu S.S., SWINNEY H.L. (1986). - Flow regimes in a circular Couette system with independently rotating cylinders. J.F.M. 164, pp. 155-183.

CHAN P.C.H., LEAL L.G. (1979). - The motion of a deformable drop in a second-order fluid. J.F.M. 92, 1, pp. 131-170.

Chandrasekhars S. (1953). - Proc. Roy. Society A, $216,293$.

CHANDRASEKHAR S. (1961). - Hydrodynamic and hydromagnetic stability: Oxford University Press. 216, 293.

ChOW A.W., Sinton S.W., IWAMIYA J.H., Stevens T.S. (1994) - Shear-indaced migration in Couetre and paralle-plate viscosimeters: NMR imaging and stress measurements Phys. Fluids.

COGNET G. (1984). - Les étapes vers la mubulence dans l'écoulement de Couette-Toylor; entre cylindres coaxiaux. J. de Méc. théo. et Appliquée, numéro spécial, p. 7 à 44 (nombreuses références bibliographiques)

Coles D. (1965). - Transition in circular Conette flow. J.F.M. 21, pp. 385-425.

COUETTE M. (1880). - Etudes sur le fiotlement des liquides. Ann. Chim. Phys. 21, p. 433-510.

DanOV K.D., IVANOV I.B., GuRKOV T.D., BORWANKar R.P. (1994). - Kinetic Model for the Simultaneous Processes of Flocculation and Coalescence in Emulsion Systems. I. of Coll. and Interface Sci. 167, pp. 8-17.

Eckstein E.C., BALley D.G., Shapiro A.H. (1977). - Selfdiffusion of paricles in shear flow of a suspension. J.F.M. 79, 1, pp. 191-208.

Elnstem A. (1906), - Amn. Phys., 19, 286 (1911). - Amm. Phys. 34,591 .

IVANOV B. (1980), - Effect of surface mobility on the dynamic behavior of thin liquid films. Pure Appl. Chem., vol. 52, p. 1241.

KIBBOUA R., LISMONDE B. (1993). - Interactions hydrodynamiques entre gouttes dans un écoulement cisaillé. $11^{\circ}$ Congrès Français de Mécanique, 6-10 septembre, Lille (France).

KIBBOUA R. (1994). - Etude d'une dispersion liquide-liquide soumise à un écoulement cisaillé simple. Caractérisation vis-à-vis de la coalescence. Thèse-Université Fourier de Grenoble.

KOCH D.L. (1989). - On hydrodynamic diffusion and drift in sheared suspensions. Phys. Fluids A 1, 10, pp. 1742-1744.

Langlois G.E., Gullberg J.E., Vermeulent T. (1954). Determination of interfacial area in instable enulsions by light transmission. Rev. Sci. Instr. 25, pp. 360-363.

LAUGHLIN C.M. Mc, RUSHTON J.H. (1973). - Interfacial areas of liquid-liquid dispersions from light transmission measurements. AIChE J., 19, pp. 817-822

LEIGHTON D., ACRIVOS A. (1987). - Measurement of shearinduced self-diffusion in concentrated suspensions of spheres, J.F.M. 177, pp. 109-131.

LEIGHTON D., ACrivos A. (1987). - The sheared-induced migration of particles in concentrated suspensions. J.F.M. 181. pp. $415-439$
LIEM A.J.S., WoODS D.R. (1974). - Review of coalescence phenomena. AlChE Symp. 70, n 144 , pp. 8-23.

LISMONDE B. (1989). - Phase distribution in developping laminar shear flow of a liquid-liquid dispersion. First liquid matter conference Lyon. Europhysics Conference abstracts-European Physical Society (1989).

MarCus P. (1984). - Simulation of Taylor-Conette flow. Part 1 : numerical methods and comparison with experiment. Part 2 : numerical results fro wavy-vortex flow with one travelling wave, J.F.M. 146, pp. 45-64 et 65-113.

MARIAUX S., ACHARD J.L. (1988). - A light transmitance probe for intefacial area measurements of dispersed wo phase flow with small particles. Experiments in Fluids, vol. 6, pp. $457-476$.

MAriauX S. (1990). - Infuence de la coalescence sur l'évolution gramulométrique des dispersions liquide-liquide dans les écoulements urbulents en conduite. Thèse de l'INP Grenoble.

MÜLLER H. (1928). - Zur allgemeinen Theorie der Reschen Koagulation. Kolloidchemische Becheft. 26, 223.

MURALIDHAR R., RAMKRISHNA D. (1986). - Analysis of droplets in turbulent liquid-liquid dispersions. Ind. Engn Fund. 25, pp. 554-560.

NADIM A. (1988), - The measurement of shear induced diffusion in concentrated suspensions with a Couette device. Phys. Fluid 31, 10, pp. 2781-2785.

NUSSENZVEIG (1965). - High frequency scatering by an impenetrable sphere. Annals of physics, vol. 34, p. 82.

NAGATA M. (1986). - Bifurcations in Conette flow between. almost co-rotating cylinders. J.F.M. 169, pp. 229-250.

NAGATA M. (1988). - On wavy instabilities of the Taylor-vortex flow between co-rotating cylinders. J.F.M. 188, pp. 585598.

Phillips R.J., ARMSTrong R.C., Brown R.A., Graham A.L., ABBOTT J.R. (1992). - A constitutive equation for concentrated suspensions that accounts for shear-induced particle migration. Phys. of Fluids A 4 (1), pp. 30-40.

RAMKRISHNA D., BORWANKER J.D. (1973). - A puristic analysis of population balance. Chem. Engn. Sci, 28, pp. 14231435.

Smoluchowski M. von (1917). - Versuch einer mahematischen Theorie der Koagulationskinetic kolloider Losungen. Z. Phys. Chem. (Leipzig) 92, 129.

STUART (1958). - On the non linear mechanics of hydrodynamic stability: J.F.M., vol. 4, pp. 1-21.

TAYLOR G.I. (1923). - Stability of a viscous liquid contained between two rotating cylinders. Phil. Trans. A 223 , pp. 289-343.

TAYLOR G.I. (1932). - The viscosity of a fluid containing small drops of another fluid. Proc. Roy. Soc. A 138, pp. 41-48.

THOMAS R.M. (1981). - Bubble coalescence in turbulent flows. Int. J. of Multiphase Flow 7, pp. 709-717.

TRAYKOV, IVANOV (1977). - Hydrodynamics of thin liquid films. Effects of surfactants on the velocity of thinning of emulsion film. Int. J. of Multiphase flow, vol. 3, pp. 471.

VAIIOULIS I.A., LIST (1984). - Collisions efficiencies of diffusing spherical particles. Adv. Colloid Interface SCI., vol. 3, p. 471 .

VETEAU J.M. (1981). - Contribution à l'étude des techniques de mesure de l'aire interfaciale dans les écoulements à bulles. Thèse USMG et INP de Grenoble.

ZINCHENKO A.Z. (1984). - Hydrodynamic interactions between two identical liquid spheres in linear flow field. Prikl. Malem. Mekhan. 47, pp. 56-63. 